\title{
DSMC Simulation of the Cometary Coma
}

\author{
Valeriy M. Tenishev and Michael R. Combi \\ Space Physics Research Laboratory, Department of Atmospheric, Oceanic and Space \\ Sciences, University of Michigan, Ann Arbor, MI 48109
}

\begin{abstract}
The study of the comet coma, or its tenuous atmosphere, is a major space application of rarefied gas dynamics, which requires modeling the gas flow in a wide range of Knudsen number. For weak to moderate comets, only the subsolar region of the coma is in a collision dominated regime. In the low density regions of the upper atmospheres of the planets and the planetary satellites and the middle to outer coma of comets the intermolecular mean free path becomes longer then the characteristic length of the problem, which makes using of conventional methods of computational gas dynamics problematic and implies the requirement to model the system based on the Boltzmann equation. Here we present results of a first application of a fully parallelized implementation of Direct Simulation Monte Carlo for axisymmetric cometary comae
\end{abstract}

\section{INTRODUCTION}

The cometary atmosphere is a unique phenomenon in the solar system. Owing to its negligible gravity, comets produce a highly variable extensive dusty atmosphere with a size much larger than the characteristic size of the cometary nucleus. As the comet approaches the sun, water vapor with some fraction of others gases sublimates, generating a cloud of gas and dust from the surface of cometary nucleus, which consists of ice and refractory materials (rocky and organic solids and dust). Sublimating gas molecules undergo frequent collisions and photochemical processes in the near nucleus region. Most of available data came from spectroscopic analysis [1] of cometary exospheres and tails due to which water vapor is considered as the major component of cometary atmosphere.

For most of the time, the gas production rate is so small that the gas flow is in a high Knudsen number regime except for the immediate regions around active areas on the sunlit nucleus. In order to model gas macroparameters of the cometary's coma the standard approaches of hydrodynamics may not be always appropriate. To get a correct description of the gas flow for given range of Knudsen numbers some methods of direct solution of the Boltzmann equation are required.

In general, solution of the Boltzmann equation, which occupies an intermediate position between the continuum theory of fluid dynamics and the discrete modeling of numerous number of particles by means of Newton's lows of classical dynamics, is a very complicated problem even for the case of a monatomic gas. The situation is usually more complicated because most of the practical applications involve consideration of polyatomic gases, which can be excited vibrationally and rotationally and for which ionization, dissociation and chemical transformations are possible.

Some methods, for which development was motivated by the need to model non-equilibrium hypersonic flows, were proposed [2] for studying such a problems. It is well known that there is no general analytical approach to solving the Boltzmann equation. Efforts were made in order to construct approximations of the solution based on different analytical techniques. The most successful examples of this approach to the problem of rarefied flow simulations are the BKW-model and expansion of velocity distribution function into a Fourier series. But the area of application for these methods is limited to simple problems. To study more realistic cases, numerical methods must be used. Among the numerical methods, which are used for solution of the Boltzmann equation, two main classes can be distinguished. In constructing the direct integration schemes, where the distribution function is approximated on a mesh in the phase space, some important physical properties of the Boltzmann equation were taken into account and were introduced into the structure of numerical algorithm, but no additional assumptions and simplifications concerning the form of the kinetic equation were made. The other approach, the direct 
simulation methods were based on imitation of molecular processes where some reasonable properties of kinetic processes were taken into account. One of the first approaches to statistical simulation was so-called the test particle method. It is based on a special iterative procedure, where the "random walk" of the test particles is studied, which interacts with the field particles. These methods are not oriented to solution of the kinetic equation but rather on modeling of intermolecular interaction and free translational motion in a gas system. There are several methods, which are based on direct simulation approach, but recently due to its simplicity and clear physical sense the DSMC method [4] has become the standard numerical method in computational rarefied gas dynamics by which other approaches are judged.

The purpose of this paper is to present results of kinetic simulation of the cometary coma. Special attention is paid to the possible outgassing of molecules which are more volatile than water. We choose $\mathrm{CO}$ as an example of an abundant volatile molecule in cometary nuclei, but other molecules such as $\mathrm{CO}_{2}$, $\mathrm{CH}_{3} \mathrm{OH}, \mathrm{H}_{2} \mathrm{CO}$, or $\mathrm{CH}_{4}$ could also be included in the model.

\section{PHYSICAL MODEL}

When the nucleus approaches the sun, it absorbs solar radiation, resulting in an increase of gas sublimation from the surface. Because the gravitational force is negligible, the gases leaving the surface form a cometary exosphere. The radiation reaching the surface and supplying energy for sublimation is partially absorbed by the dusty atmosphere. Any changes in the gas and dust production result in changes of optical characteristics of the cloud. The main volatile component of the cometary coma is water. So, water sublimation controls sublimation of others components when a comet is within about 4 astronomical units (AU) of the sun. Freshly evaporated molecules are photodissociated and photoionized, and therefore most of the chemical kinetics of cometary atmospheres involves the resulting highly reacting ions and radicals. [5] Most of the absorption of solar radiation takes place at the surface or in the dense region near the surface. Models of temperature and gas production distributions on the surface of a spherical nucleus can be found $[6,7,8]$. The simplest model of gas production assumes an optically thin coma.

The conditions for a comet coma usually consider the gas at the surface to be stationary at a given temperature. This assumption neglects the real physics of the gas surface interface. But to get correct structure of the flow field in the near nucleus region, it is more important that the flux integrated around the surface equals to desired production rate and the gas temperature near the surface is close to vaporization temperature of water. In the absence of gravity there is no downward flow and thus the resulting upwardhalf Maxwellian distribution of water vapor in a gas layer where thermodynamic equilibrium is not held. So, in a cometary atmosphere, unlike that of planets atmospheres, the mean flow velocity can be comparable with to the thermal speed [5], therefore methods adopted for studying planetary atmospheres are not longer valid. The flow then quickly thermalizes and passes smoothly through the sonic transition.

Two-dimensional axisymmetrical models for six species $\left(\mathrm{H}_{2} \mathrm{O}, \mathrm{CO}, \mathrm{OH}, \mathrm{H}_{2}, \mathrm{O}, \mathrm{H}\right)$ were considered in this work. A hard sphere collision model was assumed for intermolecular collisions. For water-water collisions the viscosity equivalent cross section [9] has been used. For other collision pairs, collision cross sections based on relevant atomic data were used [10].

The cometary nucleus was assumed to be spherical with a given radius and axisymmetrical gas emission. The gas flux from the dayside is controlled by absorption of solar radiation, and the flux varies as the square of the cosine of the subsolar angle. It was assumed that only two gaseous species sublimate from the surface: $\mathrm{H}_{2} \mathrm{O}$ and $\mathrm{CO}$. Both species are in equilibrium with the surface, and the outflow mixture is partitioned with $80 \%$ for $\mathrm{H}_{2} \mathrm{O}$ and $20 \%$ for $\mathrm{CO}$.

Photochemistry is included in the model. The main reaction branches are presented in Table 1

Table 1 Photochemical branching ratios

\begin{tabular}{|l|c|}
\hline Reaction & Branching ratio \\
\hline $\mathrm{H}_{2} \mathrm{O} \rightarrow \mathrm{H}+\mathrm{OH}$ & 0.88 \\
\hline $\mathrm{H}_{2} \mathrm{O} \rightarrow \mathrm{H}_{2}+\mathrm{O}$ & 0.22 \\
\hline $\mathrm{OH} \rightarrow \mathrm{O}+\mathrm{H}$ & 1.00 \\
\hline $\mathrm{H}_{2} \rightarrow \mathrm{H}+\mathrm{H}$ & 1.00 \\
\hline
\end{tabular}




\section{NUMERICAL METHOD}

The fundamental assumption of the DSMC [11] method is the decoupling of molecular collisions from translational motion. During a given time step, all model particles travel over the computational domain and the neighboring particles which are residents of the same collisional cell are considered as prospective partners for collision. The term "model particle" denotes a point in the phase space. After a free molecular motion, this point changes only its spatial coordinates. At the relaxation stage, a pair of model particles which occupies the same collisional cell, change their coordinates in velocity space. The conservation laws are satisfied because in every intermolecular collision the post-collisional parameters are obtained enforcing conservation of mass, momentum and energy. Such an approach results in a correct description of collisional dynamics only when translational and collisional phases are separated by the time interval $\Delta t \ll \min \left(\tau_{m}, \tau_{c}\right)$, where $\tau_{m}$ and $\tau_{c}$ are corresponding characteristic times and requires that the collisional cell small that the spatial variation is negligible. Macroscopic gas parameters, which are the moments of the distribution function, can be obtained for a given spatial cell by averaging the internal properties of all model particles in the cell. Due to the methodology, boundary conditions are stated at the level of model particles and applied each time a particle crosses the cell's boundary. A process of intermolecular collisions in the gas system is also described on the particle's level.

Various techniques have been proposed for the evaluation of the elastic collision rate. For a small time step, the number of collision pairs is small relative to the total number of model particles. So, it is good to assume that each particle can collide only once during a time step, which considerably simplifies the numerical implementation of the computational procedure. A pair of model particles from the same cell is considered for possible collision irrespective of position. The transport cross section is used in DSMC to define the local value of collisional frequency, which could cause incorrect evaluation of collision rates for processes that have cross sections close to the transport one. A pair that is selected should also be tested for chemical reactions and internal energy exchange, whose probabilities are defined as a ratio to the elastic collision probability.

\section{OPTIMIZATION TECHNIQUES}

The accuracy of the numerical results, which are obtained by the DSMC method, is very dependent on the size of sample and, finely, on the number of model particles per cell. In order to get effective use of computer resources, it is desirable to get the distribution of model particles uniformly over the domain. This is especially important at the case of 2D axisymmetrical simulation. There are several methods [12] to control the distribution of particles: variation of time steps, grid manipulation and direct variation of particles weights. The latter approach is the most widely used method in the case of axis symmetric computations: the model particle's weight is chosen proportionally to the distance from the symmetry axis, which decreases the variation of its distribution. It is possible to generalize this approach to keep the uniform distribution of model particles numbers per cell over the entire computational domain.

Due to the methodology of the DSMC method, a computational mesh is used to choose prospective collisional partners and sample the flow macroparameters. In the current studies, the problem of cometary coma dynamics was solved in an axisymmetrical geometry. The current implementation of the flow solver employs two level meshes in numerical simulation. The "background" mesh is used for definition of the geometry of the problem. To simulate interparticle collisions properly, the "background" cell can be refined to fulfill the mean free path condition for the characteristic cell size. Each "background" cell can have its own level of refinement. The mesh adaptation procedure, which is based on cell splitting, can be used to improve the quality of numerical solution and capture the macroparameter distributions in areas of high gradients. Depending on dimension of the problem to be solved, a computational mesh consists of linear segments, triangles or tetrahedrons. The derived grid is used to model relaxation processes and both of them are used for sampling the flow macroparameters.

Due to the DSMC methodology, a steady state solution can be obtained as the limit of unsteady flow simulation, which is a considerable part of the computation time. To get faster convergence, it is possible, starting with low accuracy, to improve it gradually as the current solution converges to the finial result. The two level grid approach can be used to decrease the convergence time in the case of steady state problems. In the current implementation of the flow solver, the simulation can be conducted on a set of meshes with different levels of refinement, starting with a coarse grid at the very beginning and maintaining the number of model particles per cell in desired limits. As the number of collisional cells and particles employed increase, the resolution of the computational procedure improves both in terms of flow macroparameter 
distributions and captured physical processes. Such a technique decreases the actual time of the simulation dramatically.

Parallel computers can significantly increase the range of application of the DSMC method. Effective use [13] of such computational systems can be achieved only with careful load balancing. The procedure of static load balancing, which was implemented in the code, ensures equal number of model particles among all processors used in the simulation. Such a method doesn't takes into account geometry or symmetry considerations and results in a crude work balancing. But taking into account the simplicity of the method and the fact that the initial condition, usually, is very far from the steady state, this is a good choice to balance the load at the beginning of the simulation. As the solution converges to the steady state, some corrections in the load balancing are required. A dynamic load balancing procedure is used to correct the processor load.

\section{RESULTS AND DISCUSSION}

To study influence of the gas production rate on coma structure, two different comets were used for simulations.

\section{Generic Jupiter Family Comet Validation Test}

The first set of results is for a generic weak-to-moderate production rate comet. This was done for comparison with a previous generation DSMC calculation [10]. The previous calculation was done several years ago using a typical 1995 single processor workstation $(125 \mathrm{MHz})$, and was contrasted with an Euler Equation hydrodynamic calculation. Because computer memory and time were limited, as well as having only one processor, a spherical-polar but spatially variable mesh was used and the number of cells was limited to only 2000. A comet with a production rate of $3 \times 10^{28}$ molecules was chosen, as typical of Jupiter family comets which will be spacecraft mission targets over the next decade. The radius of the nucleus was taken to be $10 \mathrm{~km}$ and the gas production rate was distributed as the square of the cosine of the subsolar angle with no gas emission on the night side. The gas was initiated at the surface at rest and with a temperature of $180 \mathrm{~K}$. The new calculations duplicate the previous physical assumptions.

The results of the calculation are shown in Figures 1 - 3. There are some differences between old and new calculations, but they are not large and are consistent with the improved spatial resolution in the new calculation, especially on the nightside, where cells in the old calculation were too large on the nightside near the symmetry axis.
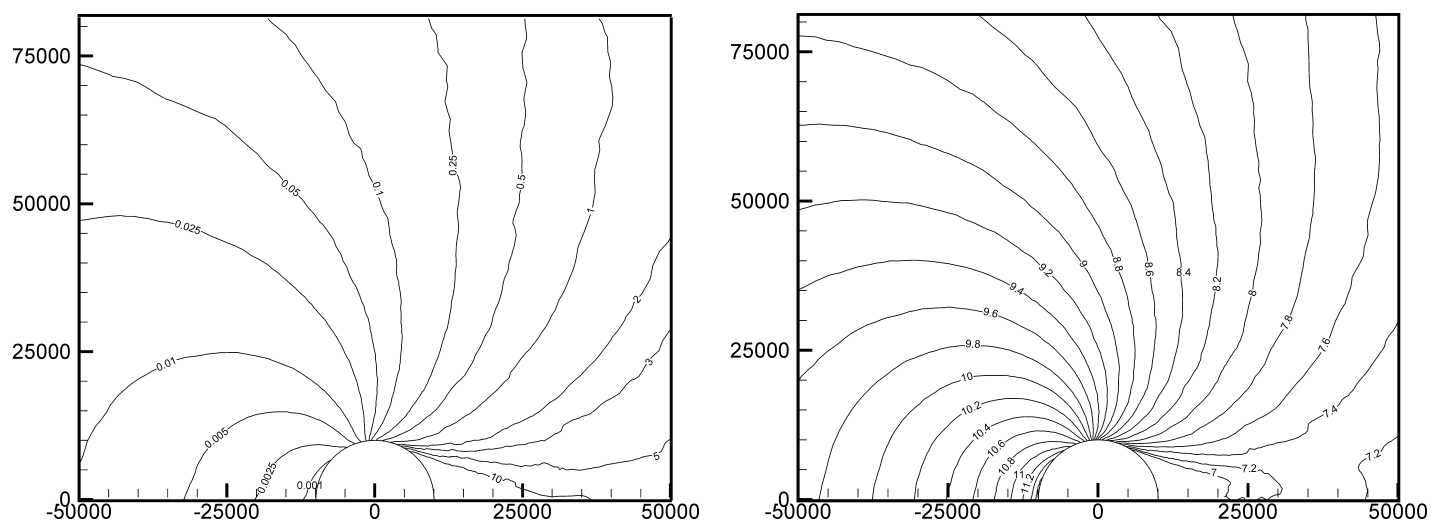

Figure 1 The two-dimensional field of Knudsen numbers and decimal logarithm of water density for the case of a generic Jupiter family comet. The coordinates on both axes are in meters from the center of nucleus in all figures. 

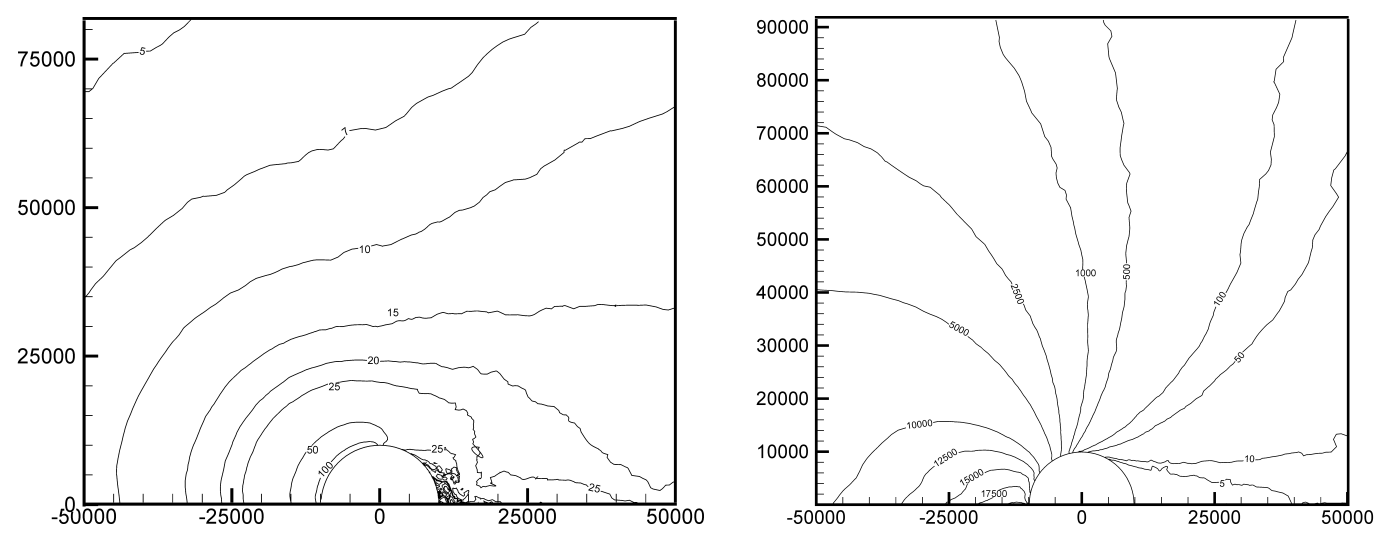

Figure 2 The two-dimensional field of temperature and Reynolds numbers for the case of a generic Jupiter family comet.
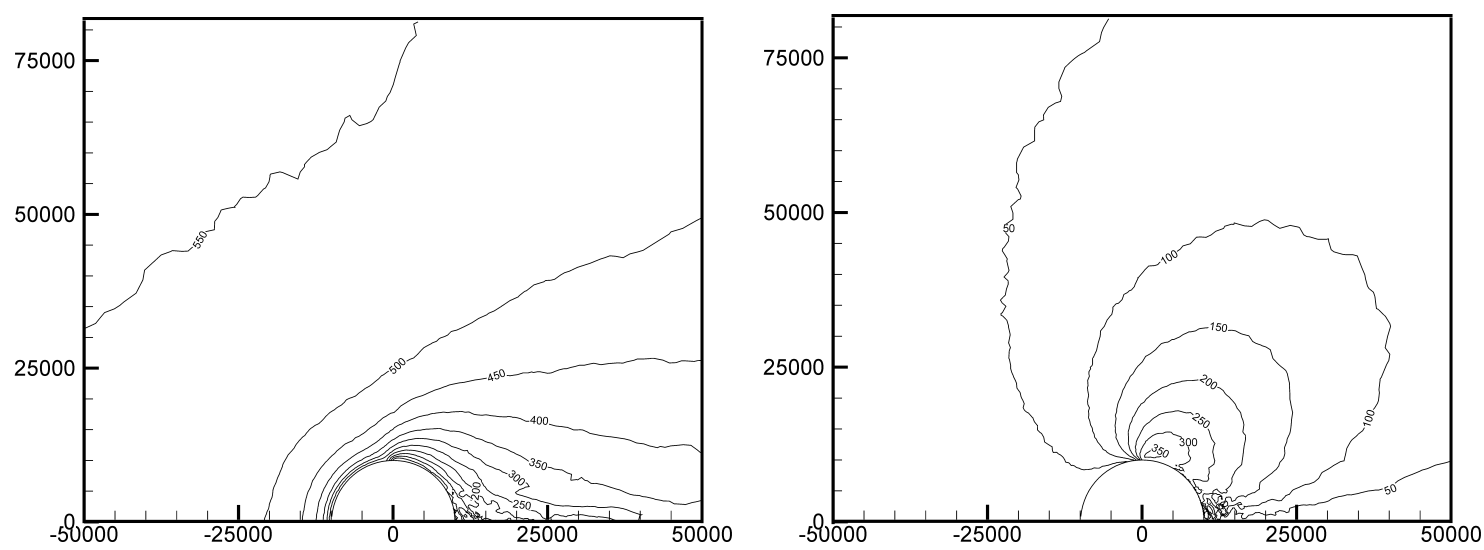

Figure 3 The two-dimensional radial and polar velocities fields for the case of a generic Jupiter family comet.

\section{Comet 46P/Wirtanen}

46P/Wirtanen is the primary target for the European Space Agency's Rosetta mission which will study the onset of cometary activity from a heliocentric distance $r=3$ AU through perihelion. In order to ensure the success of the encounter and optimize the mission parameters, it is important to determine the basic physical properties of the comet, including nucleus size and shape, rotation period. It is also important to assess the physical conditions in the coma through which the spacecraft will fly. There are some observations available of comet Wirtanen $[3,14,15,16]$ which provide an indication of the variation of the gas production rate over its orbit and an estimate of the size of the nucleus of about $0.6 \mathrm{~km}$. Here we show results for the comet at its most active at perihelion of $1.05 \mathrm{AU}$ as well as farther away at $1.5 \mathrm{AU}$.

\section{Heliocentric distance of $1.05 \mathrm{AU}$}

Similar to the previous test case, a spherical nucleus with axisymmetrical emission was considered with water production rate of $5 \times 10^{27}$ molecules pre second and surface temperature of $190 \mathrm{~K}$. In contrast to the previous case and more realistically, the whole nucleus surface is active. It was assumed that $5 \%$ of the total flux is emitted uniformly from the whole surface and the remaining $95 \%$ is emitted in a cosinesquared approximation on the dayside. Results are shown in Figures 4 - 6. 

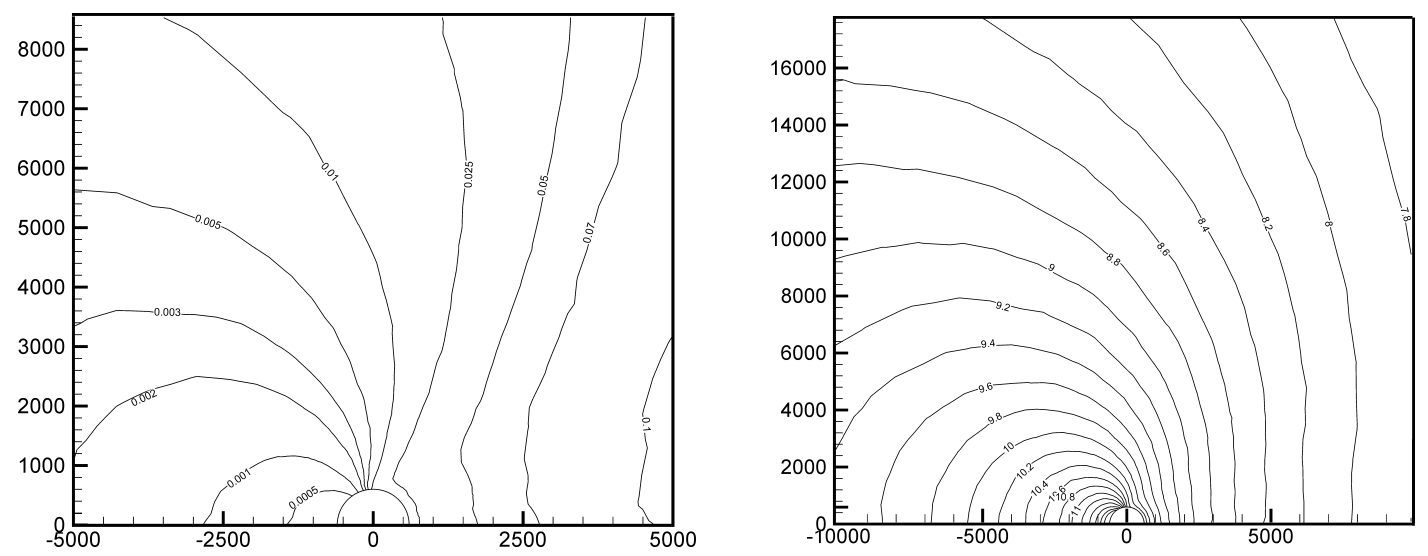

Figure 4 The two dimensional field of Knudsen numbers and decimal logarithm of water density for the case of comet Wirtanen at heliocentric distance of $1.05 \mathrm{AU}$.
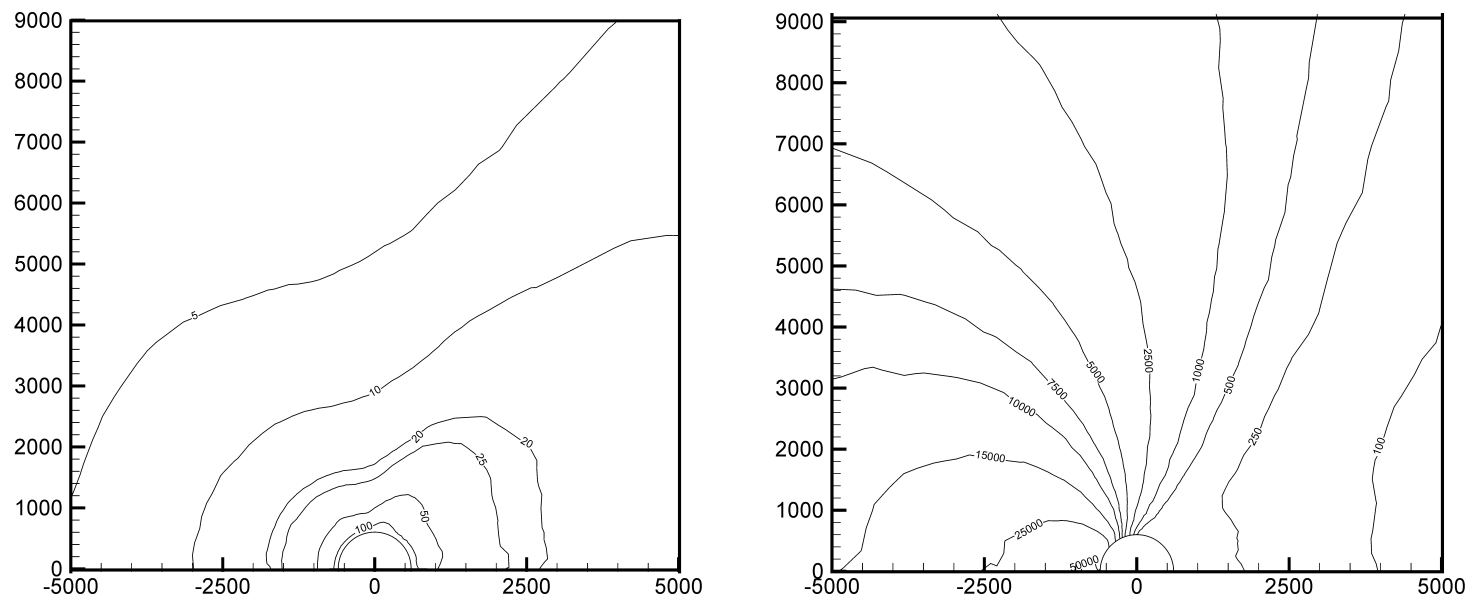

Figure 5 The two dimensional field of temperature and Reynolds numbers fro the case of comet Wirtanen at heliocentric distance of $1.05 \mathrm{AU}$.
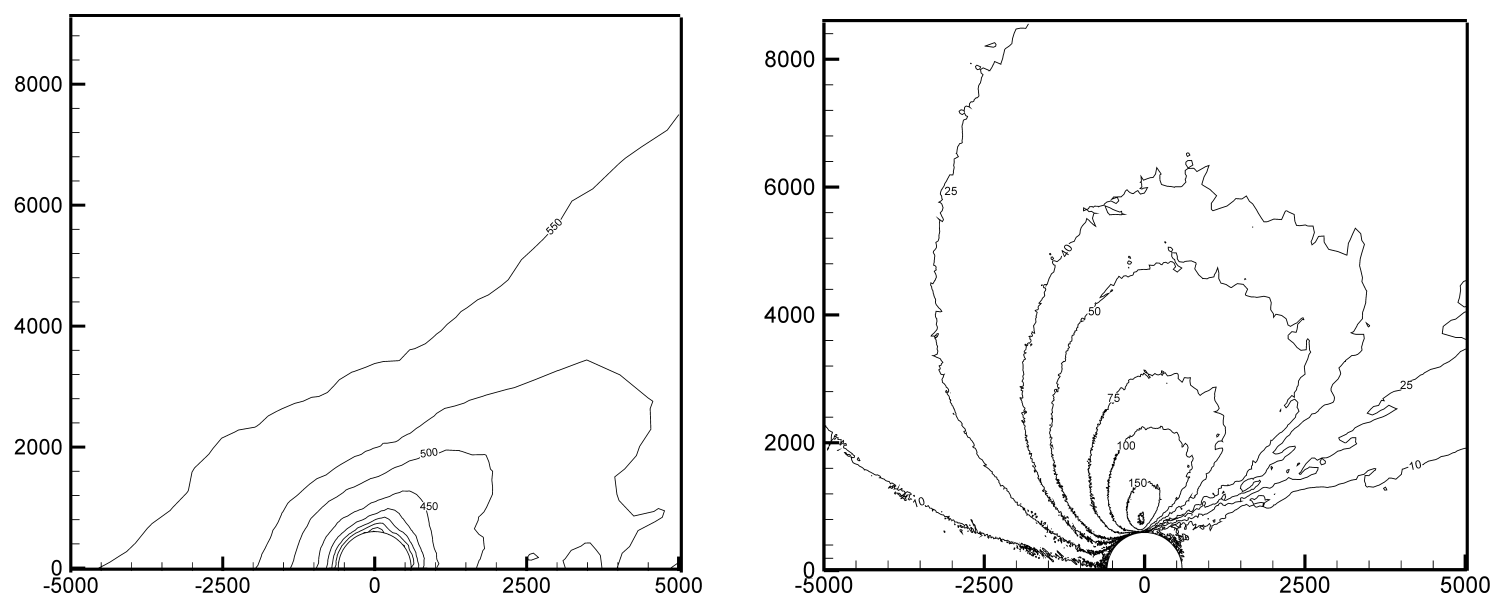

Figure 6 The two dimensional radial and polar velocities fields for the case of comet Wirtanen at heliocentric distance of $1.05 \mathrm{AU}$. 


\section{Heliocentric distance of $1.5 \mathrm{AU}$}

A similar calculation was solved for heliocentric distance of $1.5 \mathrm{AU}$, where the water production rate is only $8 \times 10^{26}$ molecules per second.
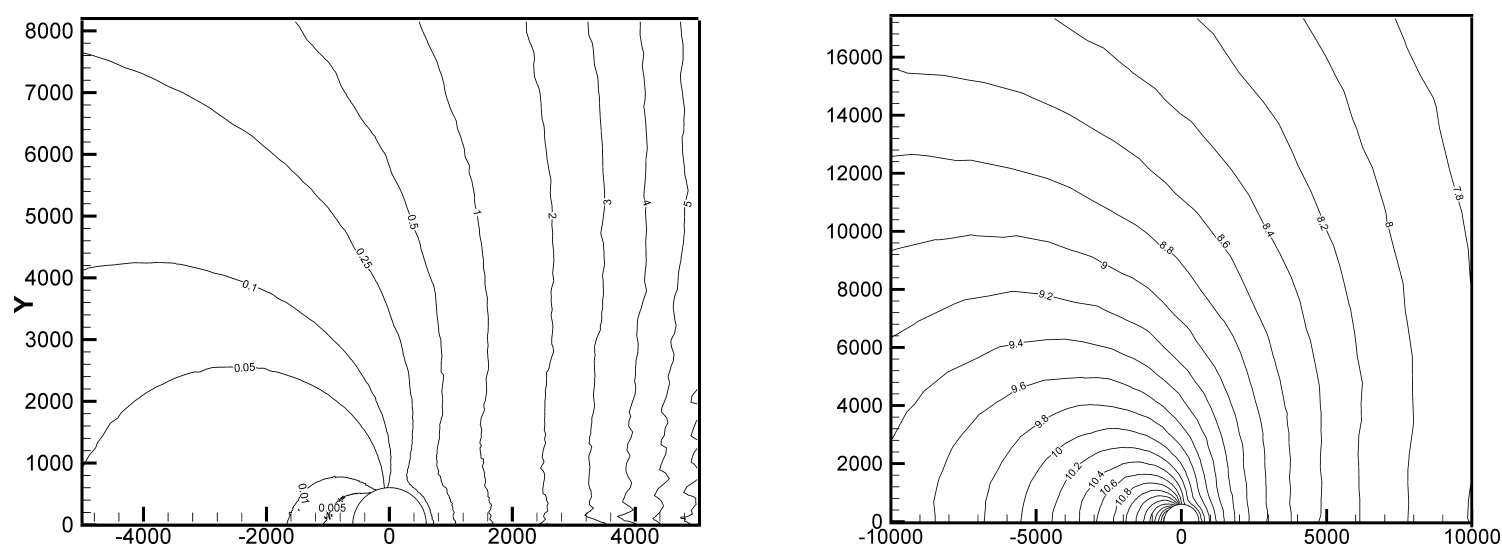

Figure 7 The two dimensional field of Knudsen numbers and decimal logarithm of water density for the case of comet Wirtanen at heliocentric distance of $1.5 \mathrm{AU}$.
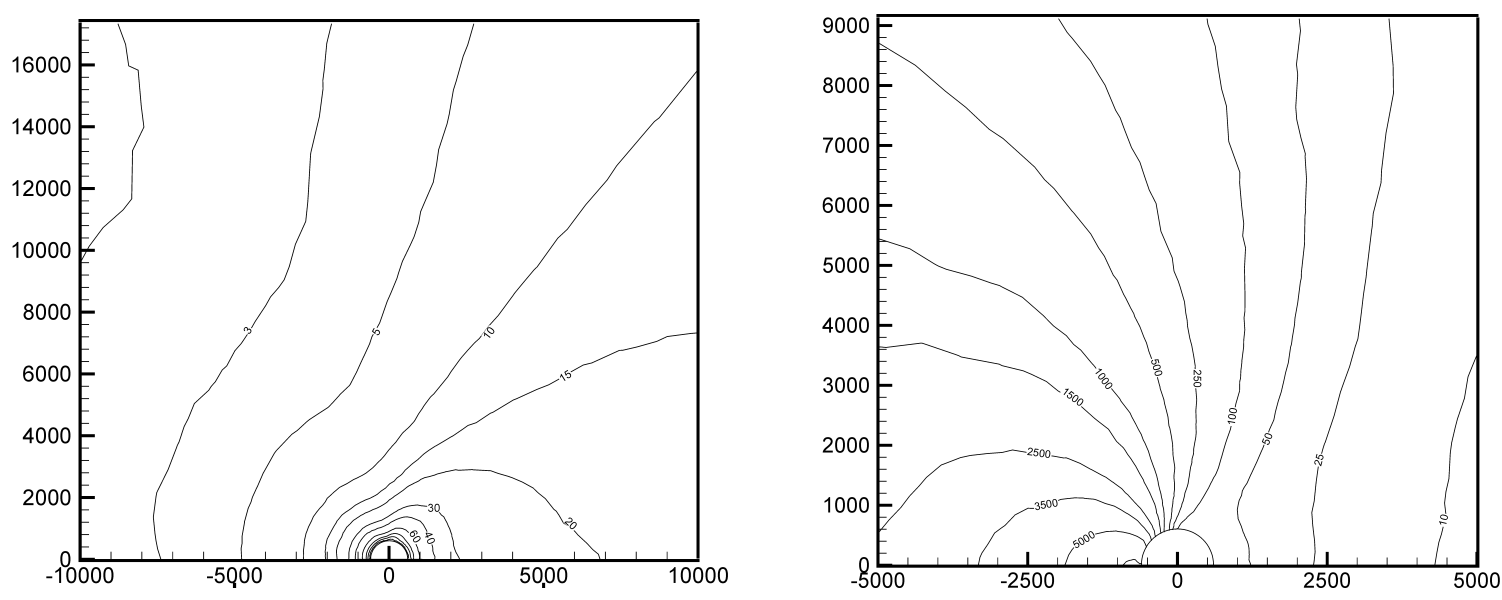

Figure 8 The two dimensional field of temperature and Reynolds numbers fro the case of comet Wirtanen at heliocentric distance of $1.5 \mathrm{AU}$.
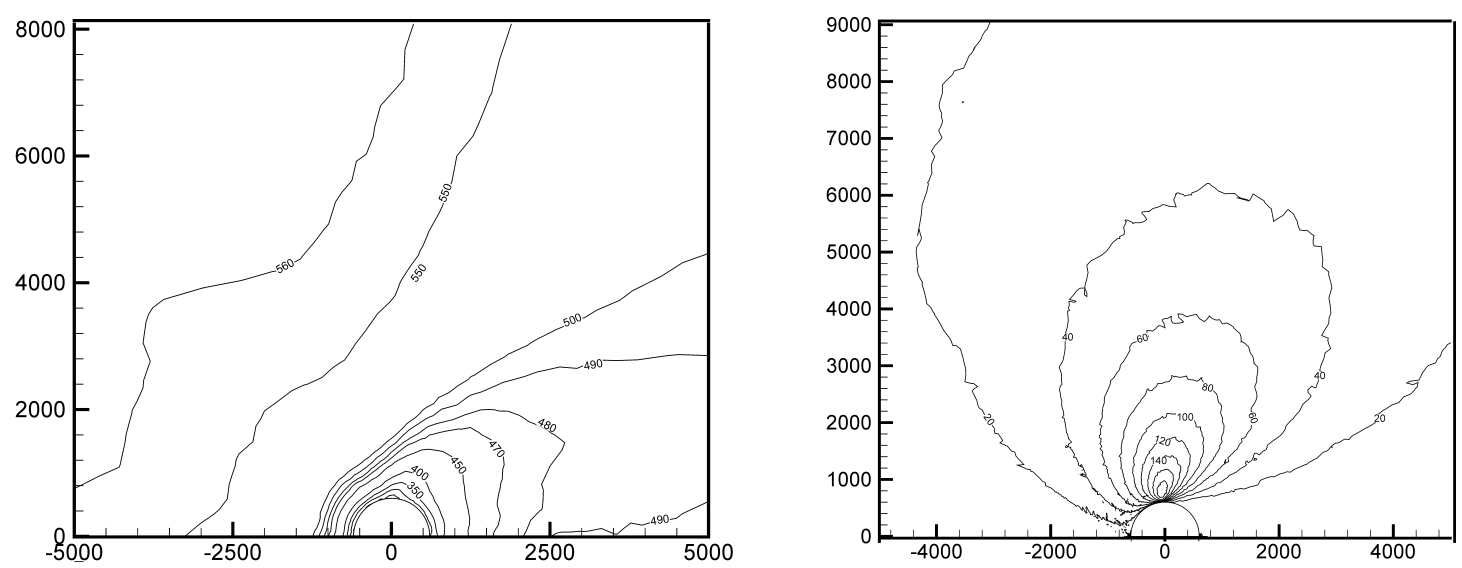

Figure 9 The two dimensional radial and polar velocities fields for the case of comet Wirtanen at heliocentric distance of $1.5 \mathrm{AU}$. 


\section{CONCLUSION}

Cometary coma simulations were performed for both weak and moderate comets to examine the influence of production rate on the structure of the coma. It is found that even with the same angular distribution of the outflowing gas, the coma surrounding the nucleus will be different in terms of distribution of the gas macroparameters such as density, mean flow velocity and temperature for different levels of overall gas production rate.

The applicability of the hydrodynamic approximation for the cometary coma was studied for different conditions as indicated from Knudsen and Reynolds number. According to the range of change of the Knudsen number, the outgassing emission flow, which is within the collision regime in the vicinity of the surface, passes through a transitional regime on the less active nightside and at the far field. So, as expected, to get the correct description of the gas flow, solutions of Boltzmann equation are required for weaker comets and less active regions around the nucleus. It was found that the hydrodynamic approximation is valid at the near nucleus region [17], and the size of this region depends on the production rate. For the stronger comet, which was considered in this study, this region spread far into and around the coma.

Periodic Comet 46P/Wirtanen is the current target of the International Rosetta Mission, which will study the onset and evolution of cometary activity. Even for the chosen heliocentric distances, at reasonable distances from the nucleus, the applicability of the hydrodynamic approximation is very questionable except for a few months on either side of perihelion. Because the production rate for comet Wirtanen is strongly dependent on the heliocentric distance and most of the time for that mission will be spent at distances even father than 1.5AU from the Sun, the hydrodynamic description of the cometary coma becomes even more problematic as a useful tool for mission planning and ultimately for data interpretation.

Direct Simulation Monte Carlo Method based flow solvers are the natural way to solve such problems.

\section{REFERENCES}

1. U. Fink, M.D. Hicks, R.A. Fevig, J. Collins, Spectroscopy of 46 P/Wirtanen during its 1997 apparition, Astron. Astrophys. 335, L37-L45 (1998)

2. V. Aristov, Direct Methods for solving the Boltzmann equation and Study of Nonequilibrium Flows, Kluwer Academic Publishers, 2001

3. P. Lamy, I. Toth, L. Jorda, H. Weaver, M. A'Hearn, The nucleus and inner coma of Comet 46P/Wirtanen, Astron. Astrophys. 335, L25-L29 (1998)

4. G. Bird, Molecular Gas Dynamics and the Direct Simulation Monte Carlo Method, Clarendon Press, 1994

5. T. Gombosi, A. Nagy, T. Cravens, Dust and Neutral Gas Modeling of the Inner Atmospheres of Comas, Rev. of Geophysics, Vol. 24, No. 3, 1086

6. J. Crifo, A. Rodionov, The Dependence of the Circumnuclear Coma Structure on the Properties of the Nucleus, Icarus 127, 319-353 (1997)

7. A. Enzian, J. Klinger, G. Schwehm, P. Weissman, Temperature and Gas Production Distributions on the Surface of a Spherical Model Comet Nucleus in the Orbit of 46P/Wirtanen, Icarus 138, 74-84 (1999)

8. P. Gutiérrez, J. Ortiz, R. Rodrigo, J. López-Moreno, A study of water production and temperatures of rotating irregularly shaped cometary nuclei, Astron. Astrophys. 355, 809-817 (2000)

9. J. Crifo, Inferences concerning water vapor viscosity and mean free path at low temperatures, Astron. Astrophys. 223, 365-368(1989)

10. M. Combi, Time Dependent Gas Kinetics in Tenuous Planetary Atmospheres: The Cometary Coma, Icarus, 123, 207-226 (1996)

11. G.A. Bird, Perception of numerical methods in rarefied gas dynamics, Progress in Astro. and Aero., 1986

12. K.C. Mannenberg, I.D. Boyd, Strategies for Efficient Particle Resolution in the Direct Simulation Monte Carlo Method, J. of Computational Physics 157:727-745, 2000

13. S. Dietrich, I. Boyd, Scalar and Parallel Optimized Implementation of the Direct Simulation Monte Carlo Method, J. Comp. Physics, 126(2):328-342, 1996

14. K. Jockers, T. Credner, T. Bonev, Water ions, dust and CN in comet 46P/Wirtanen, Astron. Astrophys. 335, L56L59 (1998)

15. S. Stern, J. Parker, M. Festou, M. A'Hearn, P. Feldman, G. Schwehm, R. Schulz, J. Bertaux, D. Slater, HST midultraviolet spectroscopy of comet 46P/Wirtanen during its approach to perihelion in 1996-1997, Astron. Astrophys. 335, L30-L36 (1998)

16. K. Meech, J. Bauer, O.Hainaut, Rotation of comet 46P/Wirtanen, Astron. Astrophys. 326, 1268-1276 (1997)

17. J. Crifo, G. Lukianov, A. Rodionov, G. Khanlarov and V. Zakharov, Comparison between Navier-Stokes and Direct Monte-Carlo Simulations of the Circumnuclear Coma. I. Homogeneous, Spherical Source, Icarus 156, 249268 (2002) 